PROCEEDINGS OF THE

AMERICAN MATHEMATICAL SOCIETY

Volume 29, Number 1, June 1971

\title{
SUMMING CLOSED $U$-SETS FOR WALSH SERIES
}

\author{
WILLIAM R. WADE
}

ABSTRACT. The union of countably many closed sets of uniqueness for the Walsh series is again a set of uniqueness.

In a classic paper on Walsh series [1] Sneider proved the finite union of closed sets of uniqueness, $E_{i}$, for the Walsh series is again a set of uniqueness. In case there were countably many sets of uniqueness he needed to further assume that $E_{i} \subset V_{i}$, where $V_{1}, \cdots$, $V_{r}, \cdots$, formed a disjoint collection of open intervals. Combining recent developments in Walsh series [2] with a slight generalization of a classical lemma (see Lemma 3) we will show this further assumption is unnecessary. The proof is similar to the proof of the trigonometric analogue given in [3].

We briefly review the definition of the Walsh functions: let $\phi_{0}(x)$ $=1$ if $0 \leqq x<\frac{1}{2}$ and $\phi_{0}(x)=-1$ if $\frac{1}{2} \leqq x \leqq 1$. Extend $\phi_{0}$ by periodicity (of period 1) to the whole real line and define $\phi_{n}(x)=\phi_{0}\left(2^{n} x\right)$. Finally, the first Walsh function is $\Psi_{0}(x) \equiv 1$, and given any $n>0$, then the $(n+1)$ th Walsh function is $\Psi_{n}(x)=\phi_{n_{1}}(x) \cdots \phi_{n_{r}}(x)$ where $n=\sum_{i=1}^{r} 2^{n_{i}}$ uniquely determines the $n_{i}$ by specifying $n_{i+1}<n_{i}$.

A set $E \subseteq[0,1]$ will be called a set of uniqueness for the Walsh series ( $U$-set for brevity) if the only Walsh series $S(x)=\sum_{k=0}^{\infty} a_{k} \Psi_{k}(x)$, satisfying

$$
\lim _{n \rightarrow \infty} S_{n}(x) \equiv \lim _{n \rightarrow \infty} \sum_{k=0}^{n-1} a_{k} \Psi_{k}(x)=0 \quad \text { for } x \notin E
$$

is the zero series.

The following lemma appeared as Theorem 4 in [1].

Lemma 1. If $S$ is a Walsh series whose coefficients, $a_{k}$, tend to zero as $k$ tends to $\infty$ and $I$ is an interval with dyadic rational endpoints then there is a Walsh series $S^{*}$ which is equiconvergent with $S$ on I and uniformly convergent to zero on $[0,1] \sim I$.

The following lemma is a corollary of Theorem 2 in [2].

Lemma 2. If $S(x)=\sum a_{k} \Psi_{k}(x)$ is a Walsh series such that

Received by the editors July 31, 1970 .

AMS 1969 subject classifications. Primary 4215; Secondary 3340.

Key words and phrases. Walsh series, set of uniqueness, of the second category on itself. 
(i) $\lim _{n \rightarrow \infty} S_{n}(x)=0$ a.e. $x \in[0,1]$;

(ii) $\lim \sup _{n \rightarrow \infty}\left|S_{n}(x)\right|<\infty$ except possibly on a countable subset of $[0,1]$;

then $a_{k}=0$ for $k=0,1,2, \cdots$.

COROLLARY. Let $E$ be a closed $U$-set contained in an open interval $J$. If $S(x)=\sum_{k=0}^{\infty} a_{k} \Psi_{k}(x)$ is a Walsh series such that

(i) $\lim \sup _{n \rightarrow \infty}\left|S_{n}(x)\right|<\infty$ for $x \in J \sim E$;

(ii) $\lim _{n \rightarrow \infty} S_{n}(x)=0$ a.e. $x \in J$;

then $S$ converges to zero for every $x \in J$.

Proof. We first notice that hypothesis (ii) implies $a_{k} \Psi_{k}(x) \rightarrow 0$ a.e. $x \in J$. But $\left|\Psi_{k}(x)\right|=1$ for any dyadic irrational $x$ so we have $a_{k} \rightarrow 0$ as $k \rightarrow \infty$. (This holds for any series satisfying (ii).)

Now $E$ is closed, so let $J_{1}$ be any subinterval of $J$ which is disjoint with $E$. Let $I$ be a subinterval of $J_{1}$ with dyadic rational endpoints. Use Lemma 1 to construct the series $S^{*}$.

Then since $I \cap E=\varnothing$, we apply Lemma 2 to $S^{*}$ to conclude $S^{*} \equiv 0$.

But $S$ and $S^{*}$ are equiconvergent, so $S$ converges to zero in $I$. Since $I$ and $J_{1}$ were arbitrary we conclude $S$ converges to zero in $J \sim E$.

Finally, if $I$ is any subinterval of $J$ with dyadic rational endpoints then applying Lemma 1 again we conclude $S^{*}$ converges to zero on $[0,1] \sim E$. But $E$ is a $U$-set so $S^{*} \equiv 0$ and thus $S$ converges to zero everywhere on $I$. But $I$ was arbitrary, so $S$ converges to zero on $J$.

Lemma 3. Let $f_{n}(n=0,1, \cdots)$ be a function which is continuous in $[0,1]$ except on a finite set $Z_{n}$. Then the set

$$
N=\left\{x: \limsup _{n \rightarrow \infty}\left|f_{n}(x)\right|=\infty\right\}
$$

is empty, countable, or of the second category on itself.

Proof.

$$
\begin{aligned}
N & =\bigcap_{k}\left\{x:\left|f_{n}(x)\right|>k \text { for some } n\right\} \\
& \equiv \underset{k}{\bigcap_{k}} .
\end{aligned}
$$

By hypothesis each $N_{k}$ is the union of an open set $V_{k}$ with a subset $Z_{k}^{*} \subseteq Z_{k}$. If $\cap V_{k}$ is empty or countable then $N \subseteq \cap V_{k} \cup \cap Z_{k}$ is empty or countable. If $\cap V_{k}$ is uncountable then it is of the second category on itself $[3$, p. 349] and since it is a subset of $N, N$ is of the second category on itself. 
TheOREM. The countable union of closed $U$-sets is a $U$-set.

Proof. Let $E_{1}, E_{2}, \cdots$ be closed $U$-sets and suppose $S$ is a Walsh series convergent to zero outside $E \equiv \bigcup_{i} E_{i}$. Šneýder [1] proved all $U$-sets have measure zero, and thus $E$ also has measure zero. In particular, $S$ converges to zero a.e.

Let $N=\left\{x: \lim \sup _{n \rightarrow \infty}\left|S_{n}(x)\right|=\infty\right\}$, and suppose $N$ is of the second category on itself.

Then by defining $N_{i}=N \cap E_{i}$ we conclude there is an open interval $J$ and index $i_{0}$ such that $N_{i_{0}} \cap J$ is dense in $N \cap J \neq \varnothing$. But $E_{i_{0}}$ is closed and $N_{i_{0}}=N \cap E_{i_{0}}$ so $N \cap J=E_{i_{0}} \cap N \cap J \subseteq E_{i_{0}} \cap J$.

We may assume the endpoints of $J$ are not in $E_{i_{0}}$, and thus that $E_{i_{0}} \cap J$ is a closed $U$-set contained in $J$. Furthermore, if $x \notin E_{i_{0}} \cap J$ then $x \notin N \cap J$ so the partial sums of $S$ are bounded in $J$ outside $E_{i_{0}} \cap J$. Thus by the corollary $S$ converges to zero everywhere in $J$. Thus $J \cap N=\varnothing$ which contradicts the choice of $J$.

Thus $N$ cannot be of the second category on itself. Lemma 3 forces $N$ to be countable or empty which by Lemma 2 implies $S \equiv 0$ as was to be shown.

\section{BiBLIOGRAPHY}

1. A. A. ŠneYder, On the uniqueness of expansions in Walsh functions, Mat. Sb. 24 (66) (1949), 279-300. (Russian) MR 11, 352.

2. W. R. Wade, $A$ uniqueness theorem for Haar and Walsh series, Trans. Amer. Math. Soc. 141 (1969), 187-194. MR 39 \#4587.

3. A. Zygmund, Trigonometrical series. Vol. 1, Cambridge Univ. Press, New York, 1959. MR 21 \#6498.

University of Tennessee, Knoxville, Tennessee 37916 